\title{
Data Rate and Bit Error Probability in Narrowband PLC Systems: OCDM versus HS-OFDM
}

\author{
Túlio F. Moreira, Ândrei Camponogara, Sobia Baig, and Moisés V. Ribeiro
}

\begin{abstract}
This study proposes a performance comparison between the orthogonal chirp division multiplexing (OCDM) and Hermitian symmetric orthogonal frequency division multiplexing (HS-OFDM) schemes in terms of data rate and bit error probability in the context of narrowband power line communication (PLC) systems. To analyze data rate of various power allocation techniques, we consider optimal, sub-optimal, and uniform power allocation techniques. Numerical results show that the OCDM eventually outperforms the HS-OFDM in terms of bit error probability when uniform power allocation applies and the HS-OFDM normalized signal to noise ratio is frequency selective. In contrast, the OCDM offers the lowest data rate performance when all the chosen power allocation techniques are considered. Thus, the OCDM can be more appropriate for low-bit-rate PLC systems when channel state information is not available at the transmitter side and fixed digital constellation is applied.
\end{abstract}

Keywords-Power line communication, orthogonal chirp division multiplexing, orthogonal frequency division multiplexing.

\section{INTRODUCTION}

The interest regarding power line communications (PLC) systems has been growing in the academic and industry spheres, mostly because of the high demand for connectivity. In fact, emerging concepts and technologies, such as Industry 4.0, smart cities, smart grids, and the Internet of Things (IoT) are directing the course of a new generation of communication systems to a scenario of reliability, flexibility, and low-power consumption. PLC technologies bring an established foundation to wired communication, since they rely on electric power grids for data communication purposes. However, electric power grids were originally meant for energy delivery purposes. Consequently, the literature highlights several impairments against the widespread use of PLC systems, such as signal attenuation with the increase of distance and frequency, multi-path effect due to impedance mismatching, load dynamics that results in impulsive noise [1]. Nevertheless, research work continues to develop techniques

This study was supported in part by Coordenação de Aperfeiçoamento de Pessoal de Nível Superior (CAPES) - finance code 001, in part by Conselho Nacional de Desenvolvimento Científico e Tecnológico (CNPq), in part by Instituto Nacional de Energia Elétrica (INERGE), and in part by Fundação de Amparo à Pesquisa do Estado de Minas Gerais (FAPEMIG).

Túlio F. Moreira, Ândrei Camponogara, and Moisés V. Ribeiro are with Electrical Engineering Department, Federal University of Juiz de Fora, Juiz de Fora, MG 36036-900 Brazil, (e-mails: \{tulio.fernandes, andrei.camponogara, mribeiro\}@engenharia.ufjf.br).

Sobia Baig is with Energy Research Center, COMSATS University Islamabad, Lahore Campus, 54000 Pakistan, (e-mail: drsobia@cuilahore.edu.pk). for improving PLC technologies and their robustness to counter such issues.

In this sense, orthogonal frequency division multiplexing (OFDM) and orthogonal chirp division multiplexing (OCDM) have proved to be useful when electric power grids are used as data communication media. OFDM is a well-established scheme, which divides a channel frequency response (CFR) into flat and orthogonal subchannels thus allowing advantage over the frequency selective channel. Regarding OFDM, [2] presented a detailed tutorial for resource allocation in PLC systems for Hermitian symmetric OFDM (HS-OFDM), which is a version of the OFDM applied to baseband communications systems. However, the OCDM scheme, which divides the data into subchirps using the Fresnel transform, is a relatively new concept for data communication and was first addressed in [3]. Regarding the baseband transmission using OCDM, [4] proposed four types of mapping schemes to transmit complex symbols through PLC channels. In addition, [5] presented a performance analysis of an OCDM scheme applied to wireless channels and compared it with the OFDM scheme. Although, all referenced works discussed here show that the OCDM scheme outperforms the OFDM scheme, mainly regarding the bit error rate (BER), still OCDM presents greater computing complexity than the OFDM [4].

Nevertheless, none of the aforementioned studies related to the OCDM scheme carried out analyses and comparisons in terms of data rate between the OCDM and OFDM schemes in the context of PLC systems. In this sense, the current study aims at analyzing and comparing the data rates related to both OCDM and HS-OFDM schemes applied to a narrowband PLC (NB-PLC) system. To do so, the optimal power allocation (OA), sub-optimal power allocation (SA), and uniform power allocation (UA) techniques are taken into account. Also, numerical results related to bit error probability, which advances the ones presented in [4], are also covered in order to bring important insights with respect to a comparative analysis between OCDM and HS-OFDM.

\section{System ModeL}

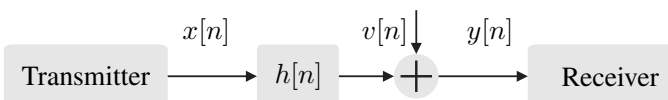

Fig. 1. Block diagram of a narrowband PLC system.

Let the block diagram showed in Fig. 1 represents a narrowband PLC system in which a PLC transmitter transmits OCDM or OFDM symbols thought a NB-PLC channel and is 


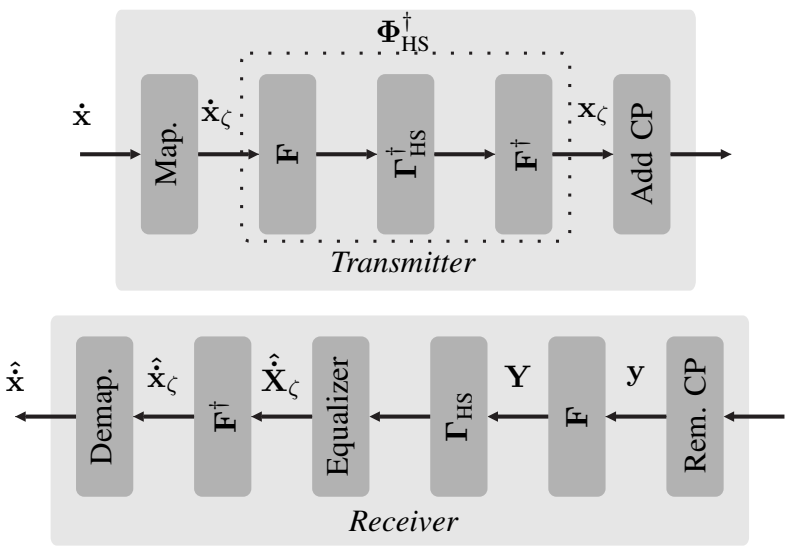

Fig. 2. Block diagram of an OCDM scheme.

demodulated and detected at the receiver. The PLC channel is assumed to be linear and time invariant since a coherence time longer than the time period associated to a $2 N$-block symbol is adopted. Also, $\{h[n]\}_{n=0}^{L-1}$ represents the channel impulse response (CIR) associated with the PLC channel, with $L$ being the CIR length, while the data-carrying signal in the discrete-time domain at the input of the receiver is expressed as

$$
y[n]=\sum_{m=-\infty}^{\infty} x[m] h[n-m]+v[n],
$$

where $\{x[n]\}$ is a sequence of $2 N$-block symbols (HS-OFDM or OCDM) and $\{v[n]\}$ is the additive noise. Note that $x[n]$ and $v[n]$ are independent and wide-sense stationary random processes, and the use of cyclic prefix (CP) avoids intersymbol interference between successive $2 N$-length symbols.

Considering NB-PLC systems, it is important to state that the channel is fading and frequency selective, and the additive noise is coloured. To address these characteristics, Subsections II-A and II-B details OCDM and HS-OFDM schemes respectively. Consequently, important aspects regarding transmission and reception in baseband as well as the derivation of the signal-to-noise ratio (SNR) equations related to both schemes are covered.

\section{A. $O C D M$}

Fig. 2 shows the block diagram of a OCDM scheme, presenting the transmitter and receiver. Since PLC systems are in the baseband, it is necessary to apply the mapping step to the modulation process to ensure that the output of the inverse discrete Fresnel transform (IDFnT) is real, i.e., $\mathbf{x}_{\zeta} \in \mathbb{R}^{2 N \times 1}$. To do so, the type IV mapping [4] is used, first extending $\dot{\mathbf{x}} \in \mathbb{C}^{N \times 1}$, which is the output of a digital modulator in the Fresnel domain, and then applying the IDFnT with the Hermitian symmetric (HS) property. Therefore, we come up with the extended symbol as

$$
\dot{x}_{\zeta}[k]= \begin{cases}\Re\{\dot{x}[k]\}, & k=0, \ldots, N-1 \\ \Im\{\dot{x}[k-N]\}, & k=N, \ldots, 2 N-1,\end{cases}
$$

where $\Re\{\cdot\}$ and $\Im\{\cdot\}$ are the real and imaginary parts of a complex signal respectively. Also, $\mathbf{x}_{\zeta}=\boldsymbol{\Phi}_{\mathrm{HS}}^{\dagger} \dot{\mathbf{x}}_{\zeta}$, where the type IV IDFnT matrix is given by $\boldsymbol{\Phi}_{\mathrm{HS}}^{\dagger}=\mathbf{F}^{\dagger} \boldsymbol{\Gamma}_{\mathrm{HS}}^{\dagger} \mathbf{F}$, with $(\cdot)^{\dagger}$ being the Hermitian operator, $\mathbf{F} \in \mathbb{C}^{2 N \times 2 N}$ represents a discrete Fourier transform (DFT) matrix, and $\boldsymbol{\Gamma}_{\mathrm{HS}} \in \mathbb{C}^{2 N \times 2 N}$ denotes a phase diagonal matrix given by

$$
\Gamma_{\mathrm{HS}}[k, k]=\left\{\begin{array}{ll}
e^{-j \frac{\pi}{2 N} k^{2}}, & \text { for } k \leq N-1 \\
e^{j \frac{\pi}{2 N} k^{2}}, & \text { for } k>N-1
\end{array} .\right.
$$

Considering the use of the CP with length $L_{c p} \geq L-1$ and perfect synchronization, the vector representation of the received symbol in the discrete-time domain can be expressed as

$$
\mathbf{y}=\mathcal{C}_{h} \mathbf{x}_{\zeta}+\mathbf{v}
$$

in which $\mathcal{C}_{h} \in \mathbb{R}^{2 N \times 2 N}$ is the circulant channel matrix associated with CIR vector $\mathbf{h} \in \mathbb{R}^{L \times 1}$, and $\mathbf{v} \in \mathbb{C}^{2 N \times 1}$ denotes the additive noise vector. To recover the transmitted information, the DFT is applied on the received signal $\mathbf{y}$ so that its output is expressed as

$$
\begin{aligned}
\mathbf{Y} & =\mathbf{F} \mathcal{C}_{h} \boldsymbol{\Phi}_{\mathrm{HS}}^{\dagger} \dot{\mathbf{x}}_{\zeta}+\mathbf{F} \mathbf{v} \\
& =\mathbf{F} \mathcal{C}_{h} \mathbf{F}^{\dagger} \mathbf{F} \boldsymbol{\Phi}_{\mathrm{HS}}^{\dagger} \mathbf{F}^{\dagger} \mathbf{F} \dot{\mathbf{x}}_{\zeta}+\mathbf{F} \mathbf{v} \\
& =\boldsymbol{\Lambda}_{H_{2 N}} \boldsymbol{\Gamma}_{\mathrm{HS}}^{\dagger} \mathbf{F} \dot{\mathbf{x}}_{\zeta}+\mathbf{F} \mathbf{v}
\end{aligned}
$$

where $\left.\boldsymbol{\Lambda}_{H_{2 N}}=\operatorname{diag}\{H[0], H[1], \ldots, H[2 N-1]]\right\}$ is the diagonal matrix of the CFR vector $\mathbf{H}=[H[0], H[1], \ldots, H[2 N-1]]^{T}$, where $[\cdot]^{T}$ is the transpose operator. Based on the commutative law of the product of two diagonal matrices, (5) can be rewritten as

$$
\mathbf{Y}=\Gamma_{\mathrm{HS}}^{\dagger} \Lambda_{\mathbf{H}_{2 \mathrm{~N}}} \mathbf{F} \dot{\mathbf{x}}_{\zeta}+\mathbf{F v} \text {. }
$$

Next, in the equalization step, we cancel out the phase induced by applying $\boldsymbol{\Gamma}_{\mathrm{HS}}$ and then we compensate $\boldsymbol{\Lambda}_{H_{2 N}}$ with a zero-forcing equalizer, in other words, $\boldsymbol{\Lambda}_{H_{2 N}}^{-1}$ is applied. Therefore, the equalized received symbol is given by

$$
\begin{aligned}
\hat{\mathbf{X}}_{\zeta} & =\boldsymbol{\Lambda}_{H_{2 N}}^{-1} \boldsymbol{\Gamma}_{\mathrm{HS}} \boldsymbol{\Gamma}_{\mathrm{HS}}^{\dagger} \boldsymbol{\Lambda}_{H_{2 N}} \mathbf{F} \dot{\mathbf{x}}_{\zeta}+\boldsymbol{\Lambda}_{H_{2 N}}^{-1} \boldsymbol{\Gamma}_{\mathrm{HS}} \boldsymbol{\Gamma}_{\mathrm{HS}}^{\dagger} \mathbf{F} \mathbf{v} \\
& =\mathbf{F} \dot{\mathbf{x}}_{\zeta}+\boldsymbol{\Lambda}_{H_{2 N}}^{-1} \boldsymbol{\Gamma}_{\mathrm{HS}} \mathbf{F} \mathbf{v}
\end{aligned}
$$

Finally, the inverse discrete Fourier transform (IDFT) is applied. As a result, the estimated OCDM block symbol is $\hat{\dot{\mathbf{x}}}_{\zeta}=\mathbf{F}^{\dagger} \hat{\mathbf{X}}_{\zeta}$. Consequently, after the demapping process, we have

$$
\hat{\dot{\mathbf{x}}}=\dot{\mathbf{x}}+\mathbf{F}^{\dagger} \boldsymbol{\Lambda}_{H_{N}}^{-1} \boldsymbol{\Gamma V}
$$

where $\boldsymbol{\Gamma} \in \mathbb{C}^{N \times 1}$ is the non-HS diagonal phase matrix with $\Gamma[k, k]=e^{-j \frac{\pi}{2 N} k^{2}}, \mathbf{F} \in \mathbb{C}^{N \times N}$, and $\left.\boldsymbol{\Lambda}_{H_{N}}=\operatorname{diag}\{H[0], H[1], \ldots, H[N-1]]\right\}$.

1) Signal to noise ratio: To analyze the SNR with a fair comparison between OCDM and HS-OFDM and to ensure uniformity in the mathematical model, we consider the received block symbol before the demapping step, i.e., $\hat{\dot{\mathbf{x}}}_{\zeta} \in \mathbb{C}^{2 N \times 1}$. In this sense, the estimated symbol in the $k^{t h}$ subchirp is given by

$$
\begin{aligned}
\hat{\dot{x}}_{\zeta}[k] & =\dot{x}_{\zeta}[k]+\frac{1}{\sqrt{2 N}} \sum_{l=0}^{2 N-1} e^{j \frac{2 \pi}{2 N} k l} e^{-j \frac{\pi}{2 N} l^{2}} H^{-1}[l] V[l] \\
& =\dot{x}_{\zeta}[k]+\dot{v}_{\zeta}[k] .
\end{aligned}
$$


According to (9), the estimated symbol has two portions. The first one is the symbol sent by the transmitter through a subchirp, $\dot{x}_{\zeta}[k]$. The second portion is the additive noise, $\dot{v}_{\zeta}[k]$. Hence, the SNR for the OCDM scheme can be expressed as

$$
\gamma_{1}[k]=\frac{\mathcal{P}_{1}[k]}{\frac{1}{2 N} \sum_{l=0}^{2 N-1}\left|H^{-1}[l]\right|^{2} \mathcal{P}_{V}[l]} .
$$

where $\mathcal{P}_{1}[k]=\mathbb{E}\left\{\dot{x}_{\zeta}[k] \dot{x}_{\zeta}^{*}[k]\right\} / 2 N \quad$ is the symbol power associated with the $k^{\text {th }}$ subchirp, $\mathbb{E}\left\{\dot{v}_{\zeta}[k] \dot{v}_{\zeta}^{*}[k]\right\}=\frac{1}{2 N} \sum_{l=0}^{2 N-1}\left|H^{-1}[l]\right|^{2} \mathbb{E}\left\{V[l] V^{*}[l]\right\}$, and $\mathcal{P}_{V}[k]=\mathbb{E}\left\{V[k] V^{*}[k]\right\} / 2 N$ is the additive noise power associated with the $k^{t h}$ subcarrier, with $\mathbb{E}\{\cdot\}$ representing the expectation operator and $(\cdot)^{*}$ being the complex conjugate operator. Observe that $V[k]$ is assumed to be a colored and stationary Gaussian random process so that $\mathbb{E}\{V[k]\}=0$ and $\mathbb{E}\left\{V[k] V^{*}[q]\right\}=\mathbb{E}\{V[k]\} \mathbb{E}\left\{V^{*}[q]\right\}, \forall k \neq q$.

\section{B. $H S-O F D M$}

Fig. 3 shows the schematic diagram of the transmitter and receiver for the HS-OFDM scheme. Note that $\mathbf{X}=[X[0], X[1], \ldots, X[N-1]]^{T}$ is the output of a digital modulator in the frequency domain. To transmit this information in baseband the HS mapping is applied as follows:

$$
X_{\mathrm{HS}}[k]= \begin{cases}\Re\{X[N-1]\}, & k=0 \\ X[k-1], & k=1, \ldots, N-1 \\ \Im\{X[N-1]\} & k=N \\ X_{i}^{*}\left[\langle-k+1\rangle_{2 N}\right] & k=N+1, \ldots, 2 N-1\end{cases}
$$

where $\langle\cdot\rangle_{2 N}$ is the circular shift operation. Considering the use of a cyclic prefix with length $L_{c p} \geq L-1$, the vector representation of the collected signal in the discrete-time domain at the receiver is given by

$$
\mathbf{y}=\mathcal{C}_{h} \mathbf{x}_{\mathrm{HS}}+\mathbf{v}
$$

where $\mathbf{x}_{\mathrm{HS}}=\mathbf{F}^{\dagger} \mathbf{X}_{\mathrm{HS}}$. Then the DFT is applied, converting the received signal (12) into the frequency domain

$$
\begin{aligned}
\mathbf{Y} & =\mathbf{F} \mathcal{C}_{h} \mathbf{x}_{\mathrm{HS}}+\mathbf{F} \mathbf{v} \\
& =\mathbf{F} \mathcal{C}_{h} \mathbf{F}^{\dagger} \mathbf{F} \mathbf{x}_{\mathrm{HS}}+\mathbf{F} \mathbf{v} \\
& =\boldsymbol{\Lambda}_{H_{2 N}} \mathbf{X}_{\mathrm{HS}}+\mathbf{V} .
\end{aligned}
$$

If zero-forcing equalization is adopted, we have

$$
\hat{\mathbf{X}}_{\mathrm{HS}}=\mathbf{X}_{\mathrm{HS}}+\boldsymbol{\Lambda}_{H_{2 N}}^{-1} \mathbf{V}
$$

Finally, the HS demapping process is applied, such that the estimation of the transmitted block symbol is given by

$$
\hat{\mathbf{X}}=\mathbf{X}+\boldsymbol{\Lambda}_{H_{N}}^{-1} \mathbf{V} \text {. }
$$

1) Signal to noise ratio: Aiming to present the SNR related to the HS-OFDM, the same approach adopted for the OCDM is considered. The received block symbol before the demapping step, from (14), the $k^{t h}$ estimated symbol is given by

$$
\begin{aligned}
\hat{X}_{\mathrm{HS}}[k] & =X_{\mathrm{HS}}[k]+H^{-1}[k] V[k] \\
& =X_{\mathrm{HS}}[k]+V_{\mathrm{HS}}[k] .
\end{aligned}
$$
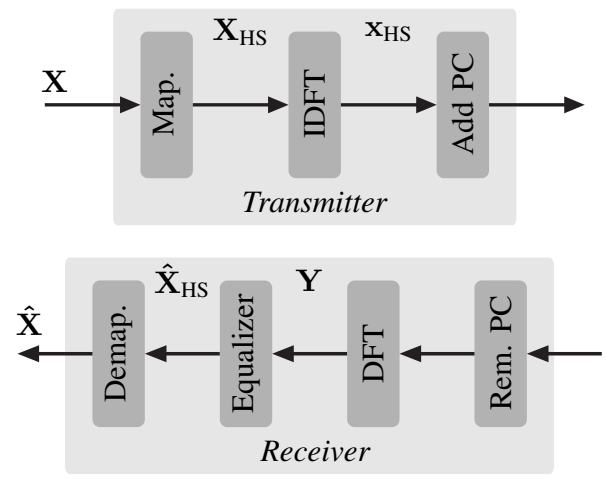

Fig. 3. Block diagram of the HS-OFDM transmitter and receiver.

Consequently, the SNR related to the $k^{t h}$ estimated symbol is given by

$$
\gamma_{2}[k]=\frac{\mathcal{P}_{2}[k]}{\left|H^{-1}[k]\right|^{2} \mathcal{P}_{V}[k]},
$$

where $\mathcal{P}_{2}[k]=\mathbb{E}\left\{X_{\mathrm{HS}}[k] X_{\mathrm{HS}}^{*}[k]\right\} / 2 N$ is the symbol power associated with the $k^{\text {th }}$ subcarrier and $\mathbb{E}\left\{V_{\mathrm{HS}}[k] V_{\mathrm{HS}}^{*}[k]\right\}=\left|H^{-1}[k]\right|^{2} \mathbb{E}\left\{V[k] V^{*}[k]\right\}$.

\section{DATA RATE AND BIT ERROR PROBABILITY}

In this section, we present mathematical expressions related to the data rate and bit error probability for the OCDM and HS-OFDM schemes. In this regard, the data rate in bits per complex symbol for both schemes can be expressed as

$$
b_{m}[k]=\log _{2}\left(1+\frac{\gamma_{m}[k]}{\Upsilon}\right)
$$

where $\gamma_{m}[k]$ is the SNR associated with the $k^{\text {th }}$ subcarrier/subchirp and the $m^{t h}$ scheme $(m=1$ for OCDM and $m=2$ for HS-OFDM), and $\Upsilon$ refers to the gap factor from Shannons capacity curve. Therefore, the data rate in bits per second (bps) can be defined as

$$
R_{m}=\frac{1}{\left(2 N+L_{c p}\right) T_{s}} \sum_{k=0}^{N-1} b_{m}[k]
$$

where $T_{s}$ is the sampling period. The total transmission power is given by

$$
\mathcal{P}_{t}=2 \sum_{k=0}^{N-1} \mathcal{P}_{m}[k]
$$

Furthermore, the upper bound for the bit error probability associated with an $M$-QAM constellation for the $k^{t h}$ subcarrier/subchirp can be expressed as

$$
P_{b, m}[k]=\frac{4}{\log _{2}(M)}\left(1-\frac{1}{\sqrt{M}}\right) Q\left(\sqrt{\frac{3}{M-1} \gamma_{m}[k]}\right)
$$

where $M$ is the constellation order. Therefore, the average bit error probability for the $m^{t h}$ scheme is given by

$$
\bar{P}_{b, m}=\frac{1}{N} \sum_{k=0}^{N-1} P_{b, m}[k] .
$$




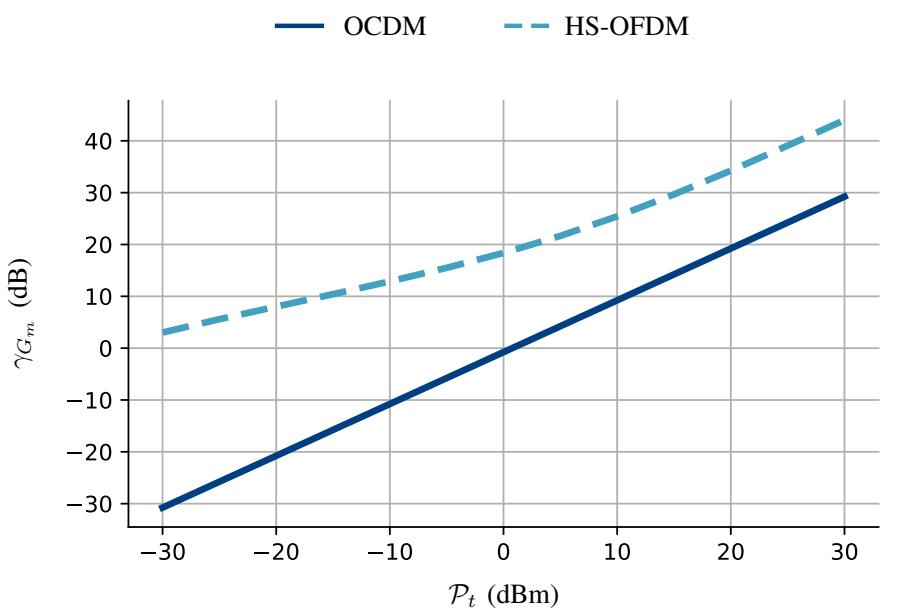

Fig. 4. SNR multichannel and multichirp, $\gamma_{G_{m}}$, in terms of the total transmission power, $\mathcal{P}_{t}$, for the OCDM and HS-OFDM schemes respectively.

\section{NumericAl RESUlts}

In this section, OCDM and HS-OFDM schemes are assessed in terms of bit error probability and data rate in the context of NB-PLC systems. Note that the 4-QAM and 16-QAM constellations are considered for the bit error probability analysis. Regarding the data rate results, we consider $\Upsilon=8.8 \mathrm{~dB}$ and the following resource allocation techniques to define $\mathcal{P}_{m}[k]$ for each subcarrier/subchirp: UA, OA (using Water-Filling algorithm with $b[k] \in \mathbb{R}^{+}$), and SA (using Levin-Campello algorithm with $b[k] \in \mathbb{N}$ ). Moreover, the bit error probability per subcarrier/subchirp as well as its average values are discussed for distinct values of $\mathcal{P}_{t}$ and under the adoption of UA. The numerical analysis are carried out considering a NB-PLC channel generated through the channel model proposed in [6] considering the parameters addressed in [7, Annex D]. Therefore, a frequency bandwidth $B=500 \mathrm{kHz}$ and $N=256$ subcarriers/subchirps are considered. The additive noise is modeled as a zero mean colored Gaussian random process with $\mathcal{P}_{V}[k]=\frac{\eta B_{s}}{2} e^{\left(-v\left|B_{s} k\right|\right)}$, where $v=1.2 \times 10^{-5}$, $\eta=1.0 \times 10^{-15}$, and $B_{s}=B / N \in \mathbb{R}$ is the sub-band frequency in Hertz (Hz) [8].

Fig. 4 shows the SNR multichannel or multichirp, $\gamma_{G, m}$, in terms of $\mathcal{P}_{t}$ for the OCDM and HS-OFDM schemes assuming UA and $\Upsilon=8.8 \mathrm{~dB}$. The SNR multichannel or multichirp can be expressed as

$$
\gamma_{G, m}=\left[\left(\prod_{k=0}^{N-1}\left[1+\frac{\gamma_{m}[k]}{\Upsilon}\right]^{1 / N}\right)-1\right] \Upsilon .
$$

Observing Fig. 4 , we see $\gamma_{G, 2}>\gamma_{G, 1}$ regardless of $\mathcal{P}_{t}$. The values vary linearly from -30.76 to $29.23 \mathrm{~dB}$ and from 3.02 to $44 \mathrm{~dB}$ for $\gamma_{G, 1}$ and $\gamma_{G, 2}$, respectively.

Regarding the performance analysis in terms of bit error probability when OCDM and HS-OFDM schemes operate with the same data rate, Fig. 5 shows the $\bar{P}_{b, m} \times \mathcal{P}_{t}$ when 4-QAM and 16-QAM constellations are considered. For an overall comparison between these schemes, we observe that HS-OFDM shows better bit error probability performance than OCDM for lower values of $\mathcal{P}_{t}$. However, as $\mathcal{P}_{t}$ increases,

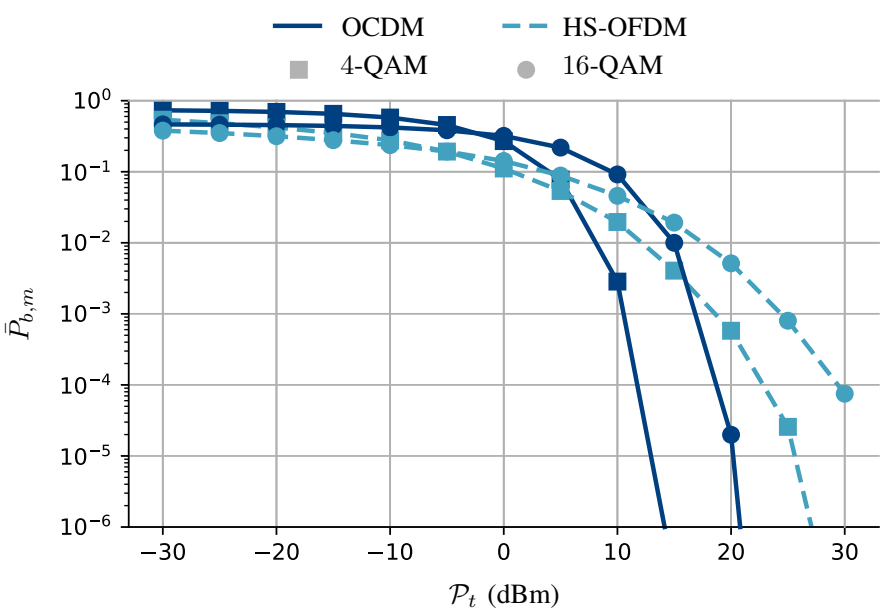

Fig. 5. Bit error probability, $\bar{P}_{b, m}$, for the OCDM and HS-OFDM schemes considering 4-QAM and 16-QAM constellations.

OCDM begins to outperform the HS-OFDM. For instance, if 4-QAM is considered, we see $\bar{P}_{b, 1}>\bar{P}_{b, 2}$ when $\mathcal{P}_{t}>6 \mathrm{dBm}$. Note that $\bar{P}_{b, 1}$ around $10^{-6}$ is found when $\mathcal{P}_{t}$ is close to $14 \mathrm{dBm}$, while $\bar{P}_{b, 2}$ with the same value is observed when $\mathcal{P}_{t}=26 \mathrm{dBm}$. In summary, the results shown in Fig. 5 corroborate the ones addressed in [4], which state that the OCDM scheme presents better performance in terms of $\bar{P}_{b, m}$ than the HS-OFDM scheme from a certain value of SNR. The discussion in the following paragraph illustrates the reason behind it.

Fig. 6 shows $P_{b, m}[k] \times k$ for OCDM and HS-OFDM schemes taking into account 4-QAM constellation and $\mathcal{P}_{t} \in\{-5,5,15\} \mathrm{dBm}$. For the OCDM, we have $P_{b, 1}[k]$ equal to $0.535,0.185$, and $1.84 \times 10^{-4}$ for all $k$ with $\mathcal{P}_{t}$ equal to $-5,5$, and $15 \mathrm{dBm}$, respectively. Meanwhile, for the HS-OFDM, $P_{b, 2}[k]$ varies in relation to $k$, reaching peak values next to $0.70,0.60,0.33$ when $\mathcal{P}_{t}$ is equal to 10 , 14 , and $20 \mathrm{~dB}$, respectively. Based on the curves showed in Fig. 6, we can see that $P_{b, 1}[k]$ shrinks as a whole as $\mathcal{P}_{t}$ increases, due to $\gamma_{1}[k]$ being constant for all $k$, while $P_{b, 2}[k]$ still present peaks of high value in specific subcarriers where $\gamma_{2}[k]$ presents a lower value due to the frequency selectively nature of the channel. In other word, under the fixed digital constellation assumption, OCDM surpasses HS-OFDM in terms of bit error probability, which makes OCDM more attractive for substituting HS-OFDM in current standards applied to NB-PLC systems in smart metering applications.

Fig. 7 shows $R_{m} \times \mathcal{P}_{t}$ for the OCDM and HS-OFDM schemes when UA, SA, and OA are adopted. In a general observation, the HS-OFDM scheme produces better results than the OCDM scheme for all resource allocation techniques. Furthermore, for the OCDM scheme, since $\gamma_{1}[k]$ is constant, the OA and UA have the same results, while the SA shows a slightly lower performance in terms of $R_{m}$. Moreover, the performance difference between the schemes are significant. In particular, adopting $\mathcal{P}_{t}=0 \mathrm{dBm}$ and UA, the OCDM and HS-OFDM reach $R_{1}=0.07 \mathrm{Mbps}$ and $R_{2}=1.66 \mathrm{Mbps}$, respectively. Furthermore, when OA is considered, the OCDM 


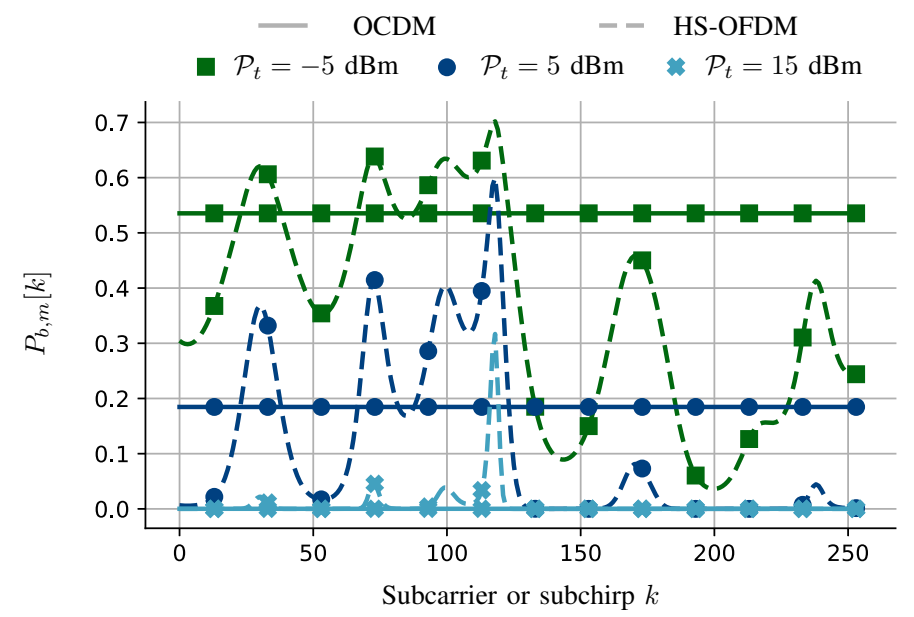

Fig. 6. Bit error probability per subchirp/subcarrier, $P_{b, m}[k]$, for the OCDM and HS-OFDM schemes considering 4-QAM.

also reaches $R_{1}=0.07 \mathrm{Mbps}$, while the HS-OFDM achieves $R_{2}=1.82 \mathrm{Mbps}$. Finally, adopting SA, $R_{1}=0.03 \mathrm{Mbps}$ and $R_{2}=1.78 \mathrm{Mbps}$ are observed for the OCDM and HS-OFDM schemes, respectively. These are direct related to the ones presented in Fig.4 where the HS-OFDM suppress the OCDM.

The OCDM scheme presents, in general, a better performance in terms of $\bar{P}_{b, m}$ because of its constant normalized signal-to-noise ratio (nSNR) for all subchirps and the adopted UA technique. Notice that such result corroborates with [9] in which the authors showed that an equal SNR for every subchannels results in an asymptotic case of the suboptimal power allocation for BER minimization. However, the same does not apply to $R_{m}$, since the HS-OFDM scheme presents better results than the OCDM scheme for all power allocation techniques considered in this paper. These variations between $\bar{P}_{b, m}$ and $R_{m}$ results can possibly be justified by distinct characteristics of $Q(\cdot)$ function used to obtain $\bar{P}_{b, m}$ and the logarithmic function used to compute $R_{m}$.

Finally, even though the OCDM scheme has shown a worse performance in terms of $R_{m}$, in practical scenarios of low-bit-rate applications, such as smart metering, the OCDM can be more suitable to implement than the HS-OFDM. Moreover, when fixed digital constellation is applied, the OCDM proves to be a better scheme due to its better results in terms of $\bar{P}_{b, m}$, which constitutes a practical scenario for NB-PLC systems.

\section{CONCLUSION}

This paper has compared the OCDM and HS-OFDM schemes in terms of bit error probability and data rate in the context of narrowband PLC systems. The numerical results have shown that the OCDM with constant nSNR brings a better performance of bit error probability compared to the HS-OFDM, assuming uniform power allocation, since the OCDM bit error probability per subchirp decreases as a whole as the total power increases, while the HS-OFDM still present peaks of bit error probability on specific subcarriers due to its variable nSNR per subcarrier. The results of bit error probability found in this study corroborates previous studies

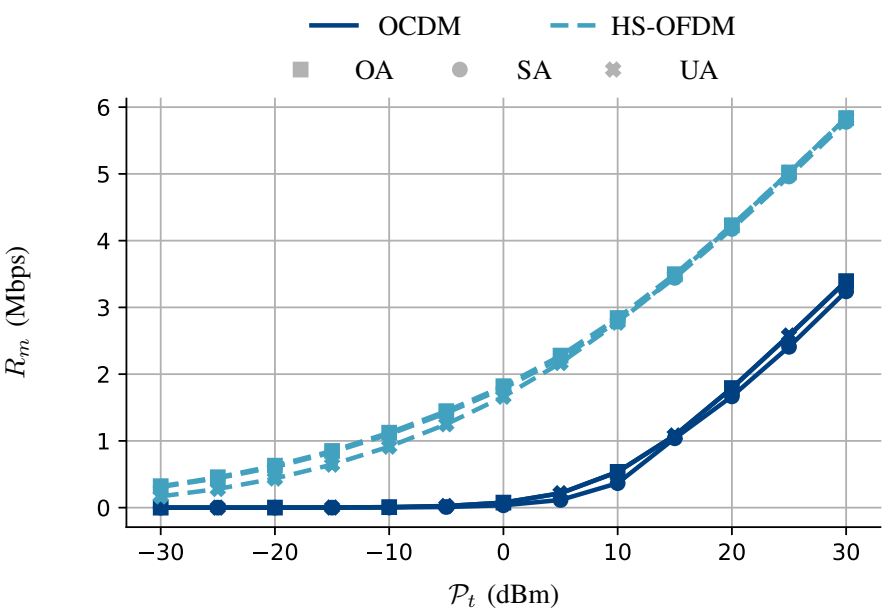

Fig. 7. Data rate, $R_{m}$, in terms of the total transmission power, $\mathcal{P}_{t}$, for the OCDM and HS-OFDM schemes considering OA, SA, and UA resource allocation techniques.

[4]. However, the HS-OFDM provides better results than the OCDM in terms of data rate for various types of power allocation techniques considered in this paper and the same can be observed with the SNR multichannel.

Therefore, the OCDM scheme proves to be a better fit for low-bit-rate applications of the PLC technology, such as smart metering, due to the better performance in terms of bit error probability.

\section{REFERENCES}

[1] T. R. Oliveira, A. A. Picorone, S. L. Netto, and M. V. Ribeiro, "Characterization of brazilian in-home power line channels for data communication," Electric Power Systems Research, vol. 150, pp. 188-197, Sep. 2017.

[2] L. G. d. Oliveira, G. R. Colen, A. J. Han Vinck, and M. V. Ribeiro, "Resource Allocation in HS-OFDM-based PLC Systems: A Tutorial," IEEE Transactions on Communications, vol. 64, no. 9, pp. 3946-3957, Out. 2018.

[3] X. Ouyang and J. Zhao, "Orthogonal chirp division multiplexing," IEEE Transactions on Communications, vol. 64, no. 9, pp. 3946-3957, Jul. 2016.

[4] L. d. M. B. A. Dib, G. R. Colen, M. L. Filomeno, and M. V. Ribeiro, "Orthogonal Chirp Division Multiplexing for Baseband Data Communication Systems," IEEE Systems Journal, vol. 14, no. 2, pp. 2164-2174, Jul. 2019.

[5] M. S. Omar and X. Ma, "Performance Analysis of OCDM for Wireless Communications," IEEE Transactions on Wireless Communications, Feb. 2021.

[6] M. Zimmerman and K. Dostert, "A multipath model for the power line channel," IEEE Transactions on Communications, vol. 50, no. 4, pp. 553-559, Apr. 2002.

[7] IEEE Standard for Low Frequency (less than $500 \mathrm{kHz}$ ) Narrow Band Power Line Communication for Smart Grid Application, IEEE Std. 1901.2, Dec. 2013.

[8] M. Katayama, T. Yamazato, and H. Okada, "A mathematical model of noise in narrowband power line communication systems," IEEE Journal on Selected Areas in Communication, vol. 24, no. 7, pp. 1267-1276, Jul. 2006.

[9] C. S. Park and K. B. Lee, "Transmit power allocation for ber performance improvement in multicarrier systems," IEEE Transactions on Communications, vol. 52, no. 10, pp. 1658-1663, Nov. 2004. 